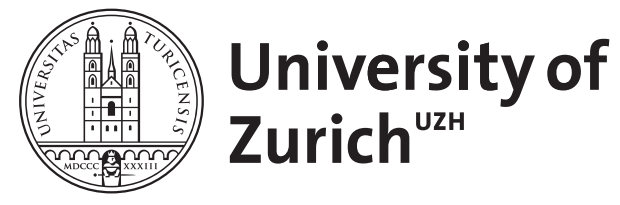

Zurich Open Repository and Archive

University of Zurich

University Library

Strickhofstrasse 39

CH-8057 Zurich

www.zora.uzh.ch

Year: 2013

\title{
Erosive Entschleunigung : Stifters Semiotisierung des Raums im Modus der
} Geologie

Frei Gerlach, Franziska

Posted at the Zurich Open Repository and Archive, University of Zurich

ZORA URL: https://doi.org/10.5167/uzh-90371

Book Section

Published Version

Originally published at:

Frei Gerlach, Franziska (2013). Erosive Entschleunigung : Stifters Semiotisierung des Raums im Modus der Geologie. In: Berbig, Roland; Göttsche, Dirk. Metropole, Provinz und Welt : Raum und Mobilität in der Literatur des Realismus. Berlin: De Gruyter, 275-289. 


\title{
Erosive Entschleunigung
}

\section{Stifters Semiotisierung des Raums im Modus der Geologie}

\author{
Franziska Frei Gerlach
}

Realistisches Erzählen bearbeitet die zeitgenössische kulturelle, ökonomische, soziale und verkehrstechnische Modernisierungserfahrung vielfach in der räumlichen Entgegensetzung von Provinz, Metropole und Welt. So sind auch Adalbert Stifters Texte weitgehend über Gegenorte organisiert, neben Stadt und Land sind das oft Wüste und Garten. Entsprechend kommt den Chronotopoi ${ }^{1}$ von Aufbruch und Rückkehr eine zentrale Stellung zu, gerade im 1857 publizierten Hauptwerk, dem Nachsommer, in dem der Bildungsweg des Protagonisten dominant über das Reisen verläuft.

Die zeittypische Beschleunigungserfahrung ist in Stifters Schreiben mit der Eisenbahn und parallel das Modernebewusstsein mit der Fabrik jedoch als Thema gegenwärtigen Romanschaffens mehr statuiert denn selbst ausgeführt. ${ }^{2}$ Vielmehr gilt das Augenmerk dem Regionalen, Herkömmlichen und den Altertümern. Es wird gesammelt, beschrieben, er-

1 Begriff und Konzept des `Chronotopos` hat Michail Bachtin in die Romananalyse eingeführt, um »[d]en grundlegenden wechselseitigen Zusammenhang der in der Literatur künstlerisch erfaßten Zeit-und-Raum-Beziehungen« zu beschreiben; siehe Michail M. Bachtin, Chronotopos. Aus dem Russischen übersetzt von Michael Dewey. Mit einem Nachwort von Michael C. Frank und Kirsten Mahlke, Berlin 2008, S. 7.

2 »Eisenbahnen und Fabriken« indizieren im Wiener Stadttext Ein Gang durch die Katakomben die Fortschrittsgläubigkeit der Jetztzeit - »in dem Jahre 1842!« - und werden mit den gröBeren Zeithorizonten der "Vorältern « und den »Jahrtausenden, [...] Jahrmillionen « und "Billionen Jahrtausende« göttlicher Planung konfrontiert; Adalbert Stifter, Wien und die Wiener. In Bildern aus dem Leben. In: Ders., Werke und Briefe. Historisch-kritische Gesamtausgabe. Hrsg. von Alfred Doppler, Wolfgang Frühwald et al., Stuttgart 1978ff., Bd. 9.1, S. 49f. Im Folgenden zitiert als $\gg \mathrm{HKG}<$ mit Bandangabe und Seitenzahl. In Stifters Briefen ist darüber hinaus dokumentiert, wie er den Bau der Eisenbahn und die Entwicklung von den Manufakturen zur Fabrik als wacher Zeitbeobachter mit verfolgt und die Transposition von »Dampfbahnen und Fabriken« in die Literatur als Einseitigkeit modernen Romanschaffens kritisch kommentiert. Vgl. Brief an Heckenast vom 22. März 1857, in: Adalbert Stifters sämmtliche Werke. 25 Bde. Hrsg. von August Sauer, Rudolf Frieb et al., Prag 1904ff., Reichenberg 1927ff. et al., Bd. 19, S. 14. Im Folgenden zitiert als `PRA mit Bandangabe und Seitenzahl. Siehe dazu Christian Begemann, Die Welt der Zeichen. Stifter-Lektuiren, Stuttgart/Weimar 1995, S. 13. 
forscht und restauriert, nicht erfunden. Und der Bildungsreisende Heinrich Drendorf ist im Nachsommer gewöhnlich zu Fuß, allenfalls noch per Donauschiff oder Kutsche, nicht aber mit der Eisenbahn unterwegs.

Das hat nicht nur mit der gewählten Route zu tun, sondern grundsätzlicher damit, dass die zentrale Zeiterfahrung Stifter'scher Texte nicht Beschleunigung, sondern Entschleunigung ist. Diese Entschleunigung hat zwar Züge einer restaurativen Moderneabwehr und bedient entsprechende Topoi, ${ }^{3}$ man wird ihr jedoch keineswegs gerecht, wenn man sie als bloße Umkehrbewegung liest. Denn die Stifter'sche Entschleunigung basiert auf einem anderen Raum-Zeit-Modell und erprobt dessen semiotische und literarische Optionen, mit denen es sich in den aktuellen kulturellen Diskurs einschreibt. Dieses andere Raum-Zeit-Modell hatte eine in ihrer Intensität der Beschleunigungserfahrung der Moderne vergleichbare grundlegende und - zumindest bei den gebildeten Zeitgenossen - auch durchschlagende Neuorientierung ausgelöst: Die Rede ist von der Geologie, die sich im 19. Jahrhundert als neue Wissenschaft etabliert hat und sogleich in den Rang einer, wie die Allgemeine Zeitung vom 13. Februar 1840 schreibt, »fashionable[n] Liebhaberei« aufgestiegen ist. ${ }^{4}$ So sollen so der wohl nicht ganz ernst gemeinte, als Zeitmarker aber aufschlussreiche Hinweis aus dem viktorianischen England - »Leute aus dem Mittelstand « in den vierziger Jahren »im allgemeinen fünf Exemplare eines teuren geologischen Werkes auf einen der beliebtesten Romane ihrer Zeit« erworben haben. ${ }^{5}$

3 Eine differenzierte und theoretisch fundierte Auseinandersetzung mit Stifters Sicht auf die Moderne, mit einem Fokus auf Urbanität, Fortschritt, Kapitalismus und Nationalstaatlichkeit, liefert Kerstin Cornils, Neues aus Arkadien. Der Streit um die Moderne bei Adalbert Stifter und Jorge Isaacs, Köln 2007. Sie bleibt aber dabei, dass Stifters Haltung eine der Moderneabwehr sei (siehe S. 10, 40), und zieht einen raumzeitlichen Perspektivenwechsel, wie ihn der vorliegende Aufsatz propagiert, nicht in Betracht.

4 Zitiert nach HKG 2.3, S. 37.

5 Diagnostiziert von der Schriftstellerin und Publizistin Harriet Martineau, zitiert nach Stephan F. Mason, Geschicbte der Naturwissenschaft, Stuttgart 1961, S. 487. Zur Entwicklung der Geologie im 19. Jahrhundert vgl. ebd., S. 466-487. Sowie grundlegend Wolf von Engelhardt, Wandlungen des Naturbildes der Geologie von der Goethezeit bis zur Gegenwart. In: Jörg Zimmermann (Hrsg.), Das Naturbild des Menschen, München 1982, S. 45-73; Martin Guntau, Die Genesis der Geologie als Wissenschaft. Studie zu den kognitiven Prozessen und gesellschaftlichen Bedingungen bei der Herausbildung der Geologie als naturwissenschaftliche Disziplin an der Wende vom 18. zum 19. Jabrbundert. In: Schriftenreibe für Geologische Wissenschaften. Berlin 22 (1984), S. 1-131. Auch für Frankreich ist belegt, dass geologische - und die davon noch nicht getrennten paläontologischen - Publikationen in den 1830er Jahren exponentiell anstiegen und an Zahl alle anderen Naturwissenschaften zusammen überstiegen; siehe Geof Bowker, Die Ursprünge von Lyells Uniformitarismus. Für eine neue Geologie. In: Michel Serres (Hrsg.), Elemente einer Geschichte der Wissenschaften. Übersetzt von Horst Brühmann, Frankfurt am Main 1998, S. 687719, hier S. 705. 


\section{Geologie: Wissensgewinn und Verunsicherung}

Geologische Erklärungsmuster faszinierten und erschütterten die Menschen im 19. Jahrhundert zugleich. Denn die Dimensionen, die sie eröffnen, tarieren Raum und Zeit radikal neu aus, verabschieden überkommenes Wissen und erschüttern existentielle Gewissheiten. Zeiträume von Milliarden von Jahren verweisen den Menschen und dessen auf 6000 Jahre veranschlagte Geschichte ins Unbedeutende, ${ }^{6}$ und das neu gewonnene Wissen vom steten Veränderungsprozess der Erdoberfläche erschüttert nicht nur das Vertrauen in die Festigkeit dieser Erdoberfläche, sondern stellt auch der Beschleunigungserfahrung der Moderne unermessliche Langsamkeit und unendliche Dauer entgegen.

Diese doppelte Bewegung der Gewinnung von Wissen und eines neuen Standpunktes einerseits, der Verunsicherung bis hin zur existentiellen Erschütterung durch die darin liegenden Gesetzmäßigkeiten andererseits, manifestiert sich in Stifters literarischer Umsetzung geologischer Narrative. Die Akkumulation von Wissen geschieht, nach dem Erarbeiten des zeitgenössischen Wissensstandes, über die geologische Feldarbeit der Protagonisten: das Sammeln von Gesteinen, Messen, Beschreiben und Abbilden von Gesteinsformationen bis hin zur Übertragung der Daten auf Karten. Paradigmatisch steht für diese Gewinnung eines neuen Standortes in der Wissensgeschichte der Gipfelblick, ${ }^{7}$ dem sich die überblickte Gegend zweidimensional erschließt und der damit die Voraussetzung für ihre kartographische Tradierbarkeit schon räumlich vorbildet:

Ich habe schon gesagt, daß ich gerne auf hohe Berge stieg, und von ihnen aus die Gegenden betrachtete. Da stellten sich nun dem geübteren Auge die bildsamen Gestalten der Erde in viel eindringlicheren Merkmalen dar, und faßten sich übersichtlicher in großen Teilen zusammen. Da öffnete sich dem Gemüthe und der Seele der Reiz des Entstehens dieser Gebilde, ihrer Falten und ihrer Erhebungen, ihres Dahinstreichens und Abweichens von einer Richtung, ihres Zusammen-

6 Eine - in Anlehnung an die Freud'sche Terminologie - »geologische Kränkung« nennt Peter Schnyder die Erschütterung, die die Entdeckung der so genannten »Tiefenzeit« und die damit verbundene Marginalisierung des Menschen und der bisherigen, christlichen Zeitrechnung bedeutete; siehe Peter Schnyder, Schrift - Bild - Sammlung - Karte. Medien geologischen Wissens in Stifters >Nachsommer. In: Michael Gamper und Karl Wagner (Hrsg.), Figuren der Übertragung. Adalbert Stifter und das Wissen seiner Zeit, Zürich 2009, S. 235-248, hier S. 235. Grundsätzlich zur »Tiefenzeit« und der von ihr ausgelösten Erschütterung von Gewissheiten siehe Stephan J. Gould, Die Entdeckung der Tiefenzeit. Zeittfeil oder Zeitsylklus in der Geschichte unserer Erde. Übersetzt von Holger Fließbach, München 1990.

7 Zum Gipfelblick in Stifters Texten siehe Sabine Schneider, Kulturerosionen. Stifters prekäre geologische Übertragungen. In: Gamper und Wagner (Hrsg.), Figuren der Übertragung (wie Anm. 6), S. 249-269, hier S. 253-259. 
strebens gegen einen Hauptpunkt und ihrer Zerstreuungen in die Fläche. (HKG 4.1$, S. 43$)^{8}$

Das geologische Untersuchungsgebiet ermöglicht die Gewinnung eines Überblicks aber nicht nur dadurch, dass es sich dem Menschen materialiter als Standort anbietet und allerorten, gerade auch an höchsten und unzugänglichsten Stellen bezwungen wird, ${ }^{9}$ sondern auch über seine Materialeigenschaften selbst.

Grundlage der Sammlung sind im Nachsommer Gesteine, die selbst schon Teil von Sammlungen waren und damit »meistens das Papierstückchen mit ihrem Namen auf sich aufgeklebt« hatten. Geordnet sind sie wie ausdrücklich bemerkt wird - nach dem maßgeblichen »Sistem von Mohs« (HKG 4.1, S. 32). Friedrich Mohs hatte in seinen Forschungen Mineralien aufgrund von Ritzproben nach ihrem Härtegrad klassifiziert und zugleich auch eine Systematik der Kristallisation entworfen. ${ }^{10}$ Entsprechend sind die erworbenen Mineralien im Nachsommer "wo möglich immer im Kristallzustande« (HKG 4.1, S. 32). Es ist materialikonographisch eine klare und stabile Struktur, die den gesuchten Überblick ermög-

8 Und weiter: »Die Betrachtung der unter mir liegenden Erde, der ich oft mehrere Stunden widmete, erhob mein Herz zu höherer Bewegung, und es erschien mir als ein würdiges Bestreben, ja als ein Bestreben, zu dem alle meine bisherigen Bemühungen nur Vorarbeiten gewesen waren, dem Entstehen dieser Erdoberfläche nachzuspüren, und durch Sammlung vieler kleiner Thatsachen an den verschiedensten Stellen sich in das große und erhabene Ganze auszubreiten, das sich unsern Blicken darstellt, wenn wir von Hochpunkt zu Hochpunkt auf unserer Erde reisen« (HKG 4.1, S. 43f.). Siehe auch HKG 4.2, S. 186: »Ich suchte auch gerne auf die Gipfel hoher Berge zu gelangen, wenn mich selbst eben meine Beschäftigung nicht dahin führte. [...] []ch sah die Länder wie eine schwache Mappe vor mir liegen.«

9 Die Suche nach der besonderen Herausforderung ist ebenso wie das Moment der Gewalt, das nicht nur das Gewinnen von Mineralien, sondern auch das Besteigen der Berge bedeutet, deutlich markiert: Höhen, die nicht zugänglich sind, werden durch »Hämmer und MeiBel zugänglich« gemacht, (HKG 4.2, S. 9), um mit so verfertigten »Leitern« auf »einen Standort« zu gelangen, »auf den zu gelangen eine Unmöglichkeit schien« (HKG 4.2, S. 186). Es wird gesucht, sich der Bergzüge »zu bemächtigen«, man geht in »die wildesten und abgelegensten Gründe [...], auf die schroffsten Grate [...]. Wie überhaupt der Mensch einen Trieb hat, die Natur zu besiegen, und sich zu ihrem Herrn zu machen, was schon die Kinder durch kleines Bauen und Zusammenfügen noch mehr aber durch Zerstören zeigen [...], so sucht auch der Bergbewohner seine Berge, die er lieb hat, zu zähmen, er sucht sie zu besteigen zu überwinden, und sucht selbst dort hinan zu klettern, wohin ihn ein weiterer wichtigerer Zweck gar nicht treibt.« (HKG 4.2, S. 185) Und als letzte Herausforderung wird all dies auch noch im tiefsten Winter gemacht, siehe HKG 4.3, S. 101-110.

10 Vgl. Friedrich Mohs, Grundriss der Mineralogie. 2 Bde., Dresden 1822-1824. Die Mohs'sche Härte-Skala von Mineralien basiert auf dem Prinzip, dass harte Stoffe weiche ritzen. Die wie eine Recherche in den Weiten des www unmissverständlich zeigt - bis heute in der Mineralogie gebräuchliche Ordinalskala ist in zehn Härtegrade eingeteilt. Mohs' Systematik kristalliner Strukturen hat sich dagegen nicht gleichermaßen durchsetzen können, hierin beruft sich die Forschung vor allem auf Christian Samuel Weiss. 
licht, und dies in doppelter Weise: Zum einen durch eine präzise definierte Klassifikation der Materialoberfläche, zum anderen durch die innere Struktur der Mineralien selbst, denn Kristalle basieren auf einer Gitterform. ${ }^{11}$

Hatte das Ersteigen eines Gipfels und die dadurch ermöglichte neue Perspektive auf das Bestehende den wissensgeschichtlichen Ort einer geologisch bedingten Neuperspektivierung in räumlicher Entsprechung abgebildet, zu dem die Mohs'sche Klassifikation und die kristalline Struktur die materiellen Entsprechungen lieferten, so steht auch für die Kehrseite des geologischen Modells von Welterkenntnis, die Erschütterung von Gewissheiten, ein genuin erdgeschichtlicher Prozess Modell: die Erosion. Auf sie kommt der Bildungsreisende bei seinen Fragen nach den Ursachen von Gesteinsformationen im Einzelnen, die sich an das Sammeln und Beschreiben des Vorgefundenen und die allgemeine Frage nach der Bildung der Erdoberfläche anschließen: Der Prozess der Erosion erweist sich als jene Gesetzmäßigkeit, die das Vorgefundene bedingt.

Diese Gesetzmäßigkeit der Erosion birgt den größten Schrecken überhaupt, gegen den die Stifter'schen Texte anschreiben und den sie zugleich anrufen: die stete und unaufhaltsame Zersetzung und letztliche Auflösung ins Indifferente, von dem her es zum Nichts nur noch ein kleiner Schritt ist. ${ }^{12}$ So stellt sich im Angesicht eines Geschiebes, einer von Stifter wiederholt beschriebenen erosiven Formation, für Heinrich alles, was an Überblick gewonnen wurde, wieder in Frage: »Wird sich vieles, wird sich alles noch einmal ganz ändern?« (HKG 4.2, S. 31)

\section{Erosion}

Es sind vor allem die Erosion und ihr Komplement, die Sedimentation, die die narrativen Modelle vorgeben, die Stifters Schreiben im Modus der Geologie organisieren. So bilden die verschiedenen Stadien der Erosion

11 Solchermaßen fest Gefügtes und still Gestelltes galt der Stifter-Rezeption lange als das Eigentliche von Stifters Schreiben und bestimmte das von der Forschung inzwischen revidierte Bild des atemporalen, im Aneinanderreihen von Dingen und Wörtern erstarrten Dichters. Dass das Starre nur eine Option unter anderen ist, zeigt sich im Nachsommer schon bei den ersten Praxisversuchen: Die Kriterien des Kristallinen erweisen sich schon bald als den Beobachtungen nicht adäquat und der Protagonist kommt nicht umhin, »sich neben den Eintheilungen, die gebräuchlich waren« seine eigenen »Beschreibungen daneben« zu machen (HKG 4.1, S. 32f.). Schneider liest dieses Vorgehen, das neben den Mineralien auch den Umgang mit Pflanzen bestimmt, als »poetologische[n] Kommentar zum Verfahren Stifter'scher Texte im Umgang mit den wissenschaftlichen Systemansprüchen seiner Zeit« und als bezeichnend für »die Ordnungsentwürfe von Stifters literarischen Universum generell« (Schneider, Kulturerosionen [wie Anm. 7], S. 249).

12 Besonders deutlich und von der Forschung entsprechend analysiert ist dies in den Weißeinbrüchen der Schneemassen in Bergkristall und Aus dem bairischen Walde. 
zusammen mit den daran beteiligten Naturkräften und dem Ausgangsmaterial selbst die Parameter für eine semiotische Ordnung, mit der der erzählte Raum deutbar wird. In diesem Sinne stellen Stein, Trümmer, Geröll, Geschiebe, Kiesel, Sand, Staub und Schichtung respektive Wetter, Wind, Wasser, Temperatur und - der persistent gemessene - Luftdruck Ordnungsraster bereit, mit dem das Spannungsfeld von Natur- und Kulturraum vermessen wird. Neben Topographien von Stadt, Land und der für Stifter typischen menschenfeindlichen Ödnis werden auch sittliche und poetologische Ordnungsanstrengungen auf eben dieser erosiven Zeichenbasis verhandelt.

Dieser narrativen Vermessung des Raums durch erosive Semiotisierung soll an exemplarischen Stellen aus dem Nachsommer nachgegangen werden. Ergänzend werden Ausschnitte aus den Bunten Steinen, den Studien, dem autobiographischen Fragment Mein Leben sowie der Textsammlung Wien und die Wiener beigezogen. Deutlich werden soll, dass die Vermittlungsstelle zwischen Geologie und Literatur in der Frage nach den zu Grunde liegenden Gesetzmäßigkeiten liegt. Dabei wird sich zeigen, dass die Verunsicherung, die das Gesetz der Erosion auslöst, auf vielfältige Entsprechungen in kulturellen wie existentiellen Fragen und darüber hinaus ins Zentrum der distinkten Zeichen selbst trifft.

Grundlage der erosiven Semiotisierung von Welt ist ein genaues Wissen vom Verlauf der Erosion und den daran beteiligten Kräften, wie sich in den Erzähltexten deutlich manifestiert. Diese exakten Beschreibungen erfüllen die realistische Intention, authentische Wirklichkeit im Text zu schaffen, indizieren aber zugleich auch Lesarten der Übertragung, insbesondere metaphorische und poetologische. Im Folgenden wird die These aufgestellt, dass in diesen Übertragungen nicht nur vom Naturmaterial inspirierte semantische, sondern auch formale Faktoren wirksam sind, die ihrerseits wiederum Sinn stiften. So dass der Eigensinn des Materials in der Sinnkonstruktion mitwirkt und neue Verbindungen motiviert. Methodisch lehnt sich dies an die in den Kunstwissenschaften entwickelte Materialikonographie an, ${ }^{13}$ die sowohl die stoffliche Beschaffenheit als auch die semantischen Kodierungen des Materials bedenkt und danach fragt, welche Aspekte davon im Text jeweils wirksam sind, umgedeutet oder narkotisiert werden. ${ }^{14}$

13 Grundlegend zur Materialikonographie der Kunstwissenschaft siehe Thomas Raff, Die Sprache der Materialien. Anleitung zu einer Ikonologie der Werkstoffe, München 1994, und Monika Wagner, Das Material der Kunst. Eine andere Gescbichte der Moderne, München 2001.

14 Diese These hat die Verfasserin an der Form des Korns - im Spannungsfeld von Sand und Getreide - bei Stifter erprobt; siehe Franziska Frei Gerlach, Die Macht der Körnlein. Stifters Sandformationen zwischen Materialität und Signifikation. In: Sabine Schneider und Barbara Hunfeld (Hrsg.), Die Dinge und die Zeichen. Dimensionen des Realistischen in der Erääbliteratur des 19. Jabrbunderts. Für Helmut Pfotenhauer, Würzburg 2008, S. 109-122. In diesem Sinne lie- 
Der Protagonist des Nachsommers, dessen erklärtes Bildungsziel ein »Wissenschafter im Allgemeinen« ist, gibt seine Studien in unterschiedlichen Wissensgebieten schon bald zugunsten der Geologie auf. Diese erscheint ihm wals Schlußstein oder Zusammenfassung aller [s]einer bisherigen Arbeiten« (HKG 4.1, S. 17, 44). ${ }^{15}$ Da sich das geologische Wissen der Zeit vor allem auf das Gebirge konzentriert, wird dieses nun sein jährliches Reise- und Bildungsziel. Dort sammelt er Gesteinsarten, beschreibt und zeichnet deren Lage, Schichtungen und die Bildungen der Erdoberfläche und findet seinen Entscheid, sich der Geologie vor allen anderen Wissenschaften zu widmen, bei der Feldarbeit bestätigt:

Wenn eine Geschichte des Nachdenkens und Forschens werth ist, so ist es die Geschichte der Erde, die ahnungsreichste, die reizendste, die es gibt, eine Geschichte, in welcher die der Menschen nur ein Einschiebsel ist, und wer weiß es, welch ein kleines, da sie von anderen Geschichten vielleicht höherer Wesen abgelöst werden kann. Die Quellen zu der Geschichte der Erde bewahrt sie selber wie in einem Schriftengewölbe in ihrem Innern auf, Quellen, die vielleicht in Millionen Urkunden niedergelegt sind, und bei denen es nur darauf ankömmt, daß wir sie lesen lernen, und sie durch Eifer und Rechthaberei nicht verfälschen. Wer wird diese Geschichte einmal klar vor Augen haben? Wird eine solche Zeit kommen, oder wird sie nur der immer ganz wissen, der sie von Ewigkeit her gewußt hat? Von solchen Fragen flüchtete ich zu den Dichtern. (HKG 4.2, S. 32f.)

Diese Reflexion versichert der Geologie ihre im Textverlauf schon wiederholt zugeordnete Gipfelstellung unter den Wissenschaften ${ }^{16}$ und macht sie im Hinblick auf ihre Funktion als Geschichte zur vielversprechendsten und reizvollsten aller möglichen Geschichten. In der doppelten Notation des Begriffs als historisch Geschehenes und literarisch zu Erzählendes bestätigen sich hier Gipfelblick und Horizonterweiterung im Modus der Literatur.

Die Korrespondenz von Geologie und Literatur verdeutlicht die Metapher vom Schriftengewölbe im Innern der Erde, in dem die Quellen zu ihrer Entzifferung aufbewahrt werden. Das ist nicht nur eine Anthropomorphisierung von Natur und rekurriert nicht nur auf den traditionalen

Ben sich die oben gemachten Bemerkungen zur kristallinen Struktur mit dem metaphorischen und poetologischen Gehalt der Stillstellungsfigur des Gitters in Beziehung bringen, der wir bei Stifter allenthalben und bis in die Streichungen in seinen Manuskripten hinein begegnen. Mit etwas anderem Akzent hat Juliane Vogel Übertragungen zwischen Wahrnehmungsphänomenen und Stifters Poetologie des Gitters untersucht: Juliane Vogel, Stifters Gitter. Poetologische Dimensionen einer Grensfigur. In: Ebd., S. 43-58.

15 Vgl. dazu auch Schneider, Kulturerosionen (wie Anm. 7), S. 251f.

16 Dass die Erdwissenschaft eine besondere Stellung unter den Wissenschaften einnimmt, wird auch von Heinrichs Mentor, dem Freiherrn von Risach, bestätigt, siehe HKG 4.1, S. 126. 
Topos vom Buch der Natur, ${ }^{17}$ sondern ist auch ein Wegweiser zu den genuin literarischen Möglichkeiten der »Gesteinwelt» (HKG 4.2, S. 185). Er führt den fragenden Drendorf direkt »zu den Dichtern« und den Nachsommer zu einer autopoetologischen Reflexion über Bedingungen und Möglichkeiten der Übertragungsleistungen zwischen den Naturgesetzen und dem Schreiben.

In der Folge berichtet Heinrich, dass zwar nach wie vor das Sammeln und fundortgetreue Abbilden des Vorgefundenen die Basis seiner Forschungen bilde, er sich aber nun stets »um die Ursachen frag[e], warum etwas sei, und um die Art, wie es seinen Anfang genommen habe. Ich baute diesen Gedanken fort, und schrieb, was durch meine Seele ging, auf. Vielleicht wird einmal in irgend einer Zukunft etwas daraus." (HKG 4.2, S. 187) Hier erweist sich explizit das geologische Gedankengebäude als Grundlage des Schreibens - auch der vorliegenden Autobiographie Heinrich Drendorfs, als die uns Stifter seinen Nachsommer präsentiert.

Dabei ist es insbesondere das Raum-Zeit-Modell, dessen Übertragung von Geologie auf Literatur augenfällig wird. Die Besonderheit geologischer Zeitlichkeit liegt - neben der genannten Langsamkeit und Dauer darin, dass sie sich räumlich manifestiert: Zeit ist als Schichtung, als Ansammlung von Sedimenten ablesbar und wird über deren Einschlüsse, die Geschichten von längst untergegangenen Zeiten erzählen können, datierbar. ${ }^{18}$ Eine literarische Transformation von Geologie bedeutet also eine Verräumlichung von Zeit. Diese Dominanz des Räumlichen beim Erzählen von Zeitabläufen ist im Nachsommer im zyklischen Ablauf der - durch den Romantitel ja bedeutsamen - Jahreszeiten präsent. Ihr Wechsel ist räumlich gefasst und wird durch die jahreszeitlich wechselnden Aufenthaltsorte des Protagonisten angezeigt. Dies geht im Detail so weit, dass der Zeitpunkt der Rosenblüte, der das raumzeitliche Zentrum des Romans bildet, für das Gebirgstal, die Metropole und die Landgüter im voralpinen Raum jeweils exakt angegeben wird.

Zeit wird gemäß den geologischen Ordnungskriterien im Nachsommer aber nicht nur im Allgemeinen räumlich kodiert, im Modell der Schichtung wird im Besonderen der Menschheitsgeschichte ein spezifischer ZeitOrt zugewiesen: der eines »Einschiebsel[s]«, und zwar eines besonders kleinen. Die Zeit menschlicher Geschichte als kleines »Einschiebsel« zu bezeichnen, bedeutet eine radikale Dezentrierung des Menschen. Der Index des Kleinen, der dem Einschiebsel zugeordnet ist, meint in Stifter'schem Deutungskontext nun aber gerade nicht das Unbedeutende.

17 Vgl. dazu Schnyder, Schrift (wie Anm. 6), S. 240f.

18 Indiziert ist dies im Nachsommer in der Inszenierung fossiler »Amoniten« in »der Mitte« des Marmorsaals, des ikonographischen Zentrums des Rosenhauses, und damit überdeterminiert zentral, vgl. HKG 4.1, S. 81; dazu Schnyder, Scbrift (wie Anm. 6), S. $245 f$. 
Vielmehr liegen im Kleinen und Kleinsten die Grundlagen von Bedeutungsstiftung und werden Regeln von Gesetzmäßigkeiten ablesbar. ${ }^{19}$

\section{Sand und Staub}

Programmtisch hat Stifter das in seiner viel zitierten Formulierung vom »sanfte[n] Gesez« (HKG 2.2, S. 12) in der Vorrede zur Erzählsammlung der Bunten Steine von 1853 formuliert, in der er das Gesetz der Gerechtigkeit, der Sitte und der gegenseitigen Anerkennung und Liebe über eine Neubesetzung von Großem und Kleinem entwickelt. Erklärtes Ziel ist dabei, »ein Körnlein Gutes zu dem Baue des Ewigen beizutragen« und so eine Spur vorzugeben, auf der »Körnchen« an »Körnchen« gereiht werden könne (HKG 2.2, S. 9f., 11). Geht es Stifter hier erklärtermaßen um eine sittliche Ordnungsanstrengung, so greift er die Körnchenmetaphorik im autobiographischen Fragment Mein Leben von 1867 in einem deutlicher ins Poetologische gewendeten Sinn wieder auf und leitet seine Erinnerungserzählung vom Eintritt in die symbolische Ordnung und seine Selbstvergewisserung im Schreiben darüber mit einer Hommage an das kleinste Sandkörnchen ein:

Es ist das kleinste Sandkörnchen ein Wunder, das wir nicht ergründen können. $\mathrm{Da}$ es ist, daß seine Theile zusammen hängen, daß sie getrennt werden können, daß sie wieder Körner sind, daß die Theilung fort gesezt werden kann, und wie weit, wird uns hienieden immer ein Geheimniß bleiben. [...] Wir stehen vor dem Abgrunde dieses Räthsels in Staunen und Ohnmacht. (PRA 25, S. 176) ${ }^{20}$

Nicht von ungefähr sind es beide Male geologische Kontexte, in denen die Wertigkeit des Kleinen entwickelt wird: Es ist explizit und implizit der Prozess der Erosion, der für das Entstehen der Körnchen verantwortlich ist. Explizit benennt das autobiographische Fragment die "großen Körper, davon« das Sandkörnchen »getrennt worden ist, und die den Außenbau unserer Erde bilden« (PRA 25, S. 176), und also den Herkunftsort im Prozess der Erosion und fragt darüber hinaus nach deren Fortgang: Die Frage nach der weiteren Teilbarkeit und deren potentiellem Ende führt

19 Paradigmatisch zeigt sich dies bei der Lösung des Wetter-Rätsels im ersten Buch des Nachsommers. Während Heinrichs Messgeräte und sein auf Wanderungen gewonnenes meteorologisches Erfahrungswissen ein Gewitter anzeigen und ihn dazu veranlassen, um Einkehr im Rosenhaus zu fragen, basiert Risachs gegenteilige und sich schließlich bewahrheitende Prognose auf der langjährigen Beobachtung der »Insekten« und »kleinen Thiere«: Aus ihrem Verhalten lassen sich die zuverlässigsten Prognosen ableiten, wie überhaupt die »kleinsten Dinge« die großen Gesetzmäßigkeiten bergen (HKG 4.1, S. 121-125).

20 In einer späteren Fassung lautet die entsprechende Stelle dann: »edes Sandkorn ist ein Ding, dessen Wesenheit wir nicht ergründen können.« (PRA 25, S. 181) 
geologisch zu einer Korngröße von unter $0,063 \mathrm{~mm}$, dem minimalen Durchmesser eines Sandkorns, und damit zum Staub, ${ }^{21}$ metaphorisch hingegen in die Bedeutungslosigkeit und Unlesbarkeit der Zeichen.

Damit droht im Wirken des Naturgesetzes der Erosion, in dessen Rahmen die Sprachgewinnung im autobiographischen Fragment gestellt wird, gerade der Verlust des Gewonnenen. Doch auch das Entstehen von Bedeutung über das Schaffen von Differenzen vollzieht sich keineswegs sanft, sondern wird als schmerzhafte Gewalt- und Trennungserfahrung erzählt, 22 die sich wiederum auf den Erosionsprozess selbst abbilden lässt. Es sind in diesem Sinne nicht nur die semantischen Felder aus der Geologie und der Autobiographie eines Schriftstellers, die in ein Entsprechungsverhältnis gebracht werden und über gegenseitige Übertragungen einen Mehrwert an Sinn eröffnen, sondern es sind strukturell gesehen die Gesetzmäßigkeiten selbst, die ein formales Korrespondenzverhältnis zwischen Geologie und Semiotik ermöglichen und damit eine tiefgründige Übertragungslogik anstoßen.

Anders als im autobiographischen Fragment ist der Erosionsprozess in der "Vorrede« der Bunten Steine trotz der explizit geologischen Titelgebung nur implizit präsent, er aktualisiert aber auch hier einen gewaltbesetzten Subtext. In seiner Aufzählung dessen, was Stifter für wahrhaft groß hält, nennt er unter anderem »[d]as Wehen der Luft das Rieseln des Wassers [...] das Wogen des Meeres [...] das Glänzen des Himmels" (HKG 2.2, S. 10) und damit also Kräfte und Einflüsse, die die Zerstückelung, Schleifung und Vertreibung des Ursprungsmaterials hin zum Körnchen im erosiven Prozess steuern. Liest man die Isotopie der Erosion als Index des sanften Gesetzes mit, so schreibt sich auch hier ein Gegensinn von Gewalt und Trennung ein, der die Sanftheit der kleinen Bewegung und der Hinwendung zum Kleinen und Kleinsten hintertreibt. ${ }^{23}$

Diese Signatur von Gewalt manifestiert sich im Nachsommer in einer mehrseitigen Reflexion über die Erosion als Anhäufung sich immer weiter

21 Vgl. Art. sSand in: Lexikon der Geowissenschaften in sechs Bänden. Hrsg. von Christiane Martin, Manfred Eiblmaier et al., Heidelberg/Berlin 2000-2002, Bd. 4, S. 366.

22 Ausgehend von einer Ganzheitserfahrung bilden sich die »Außenwelt« als eine davon unterschiedene Wahrnehmung sowie die »Gestalt« der Mutter heraus, die Fähigkeit zu unterscheiden setzt ein und mit ihr das Sein in der symbolischen Ordnung. Diese Schwellenerfahrung ist an eine Gewalterinnerung geknüpft: an »Klingen, Verwirrung, Schmerz in meinen Händen und Blut daran«. Diese einschneidende Erinnerung ist einerseits verbunden mit der »Erleichterung, die alle Male auf das Weichen des Entsezlichen und Zugrunderichtenden folgte«, die im »ich sagte: `Mutter, da wächst ein Kornhalm« sprachlich wird. Andererseits verbindet sich damit die ebenso unauslöschliche Erfahrung einer doppelten Nicht-Anerkennung des Ichs: »Die Großmutter antwortete darauf: `Mit einem Knaben, der die Fenster zerschlagen hat, redet man nicht«, und »die Mutter sprach wirklich kein Wort» (PRA 25, S. 178f.).

23 Differenziert dazu Frei Gerlach, Die Macht der Körnlein (wie Anm. 14), S. 117-120. 
ausdifferenzierender Fragen, die sprachlich quasi mimetisch umsetzt, was die Erosion im Prozess ihres steten Aufteilens und Aufsplitterns und Neuordnens am Stein vollzieht:

\begin{abstract}
Wird sich vieles, wird sich alles noch einmal ganz ändern? In welch schneller Folge geht es? Wenn durch das Wirken des Himmels und seiner Gewässer das Gebirge beständig zerbröckelt wird, wenn die Trümmer herabfallen, wenn sie weiter zerklüftet werden, und der Strom sie endlich als Sand und Geschiebe in die Niederungen hinausführt, wie weit wird das kommen? Hat es schon lange gedauert? Unermeßliche Schichten von Geschieben in ebenen Ländern bejahen es. Wird es noch lange dauern? So lange Luft Licht Wärme und Wasser dieselben bleiben, so lange es Höhen gibt, so lange wird es dauern. Werden die Gebirge also einstens verschwunden sein? Werden nur flache unbedeutende Höhen und Hügel die Ebenen unterbrechen, und werden selbst diese auseinander gewaschen werden? (HKG 4.2, S. 31)
\end{abstract}

Erosion zeigt sich bei Stifter als ein radikaler und gewaltsamer Prozess, dem durch die lange Dauer, die Langsamkeit und die Körnchengestalt eine Signatur des Sanften anhaftet. In der Modellierungsleistung der Erosion für die Literatur wirkt sich beides aus. In diesem Sinne transportieren Kiesel oder Sand auch den Gewaltaspekt, der ihrer Genese inhärent ist, als materielles Gedächtnis mit und wirken als latenter Subtext auf den - bei Stifter topischen - rein gefegten Sandwegen in wohl geordneten Gartenlandschaften.

Das zeigt sich besonders eindrücklich in der 1842 erstmals erschienen Erzählung Abdias, bei der die afrikanische Wüste und ein Garten im mitteleuropäisch-voralpinen Raum über den Sand in ein Korrespondenzverhältnis treten. In einer halb im Sand versunkenen, waus der Geschichte verlorenen Römerstadt« (HKG, 1.5, S. 239) fristet Abdias eine Existenz ganz im Zeichen der Erosion: Es versanden die Seelen-, Sozial- und Kulturlandschaft gleichermaßen. Kultur ist als Prozess der Zersetzung und Anhäufung kontingenter Kulturfragmente und also als Kulturerosion gestaltet. Semiotisch erweist sich die leere Fläche des Sandes, hier einer langen Tradition folgend, zwar als ein poetologisches Reflexionsmedium, doch nicht im positiven Sinn: Die eingeschriebenen Zeichen sind flüchtig und durch die Irritation des Flimmerns unlesbar. Der Auszug aus dem Sandland bringt die erwartete Erlösung nicht, der zerstörerische Subtext fährt aus der afrikanischen Wüste ins mitteleuropäische Getreideanbaugebiet mit, und in einer für Stifter typischen metonymischen Engführung von Sand- und Getreidekorn bringt letzteres auch im scheinbar idyllischen Gegenort nur Zerstörung. ${ }^{24}$

24 Dies ist ausgeführt in Frei Gerlach, Die Macht der Körnlein (wie Anm. 14), S. 112-117. 
Stifters Sandformationen und ihrem Weg zwischen Natur- und Kulturzeichen ließe sich noch in viele Texte und Orte folgen, namentlich in die Karlandschaft von Kalkstein (1853), nicht aber in die Stadt. In Stifters erosiver Semiotisierung des Raums ist es nicht die gleichförmige Masse des Sandes, sondern das ungefüge Geschiebe, das den Raum der Stadt und darin insbesondere die Menschen selbst bezeichnet.

\section{Menschliche Geschiebe}

Einen solchen »Haufen von Geschiebe $[\ldots]$ aus einem Flußbette«, das seiner Verwendung zum Straßenbau am Rand der Metropole harrt, betrachtet der ınachsommerlich« Reisende mit »Ehrfurcht«:

Ich erkannte in den rothen weißen grauen schwarzgelben und gesprenkelten Steinen, welche lauter plattgerundete Gestalten hatten, die Bothen von unserem Gebirge, ich erkannte jeden aus seiner Felsenstadt, von der er sich losgetrennt hatte, und von der er ausgesendet worden war. Hier lag er unter Kameraden, deren Geburtstätte oft viele Meilen von der seinigen entfernt ist, alle waren sie an Gestalt gleich geworden, und alle harrten, daß sie zerschlagen und zu der Straße verwendet würden. (HKG 4.2, S. 27f.)

Erosion mit ihren Stadien der Abtrennung und der Wegführung wird hier enggeführt mit dem Lebensweg jener Menschen, die, in der Provinz geboren, von dort ausziehen und der Stadt zustreben. Ein ganzer Narrationsplan ist darin angelegt, und mit ihm auch das absehbare Ende: der Verlust der Individualität, die Anpassung an die Masse, das passive Verharren im Erwarten des Endes, die Zerschlagung der Ambitionen noch vor Erreichen der Metropole und die Transformation eines jeden in ein Stückchen Wegs, auf dem die später Kommenden weiter gehen.

Wie ein Komplement zu dieser vom Stein ausgehenden Übertragung liest sich eine von der sozialen Masse ausgehende Beschreibung in Aussicht und Betrachtungen von der Spitze des St. Stephansthurmes aus der Erzählsammlung Wien und die Wiener von 1844, die Stifter als Herausgeber betreute und zu der er auch eigene Beiträge beisteuerte: Die »Masse der Vorstädte«, so heißt es da, schiebt sich gegen die Stadtgrenze heran, am »Glacis«, der einstigen Stadtbefestigung, "gleichsam in ihrem Hereinschieben gegen die Stadt hier an einer unsichtbaren Grenze anhaltend und sich anstaunend« (HKG 9.1, S. X). Das Glacis, jetzt ein »grüner Gürtek, hält in seinem Wortstamm auch jenes Isotop des Glacialen präsent, das das soziale Geschiebe der Vorstädte als Gletschermoräne zu seinem Ende kommen lässt. Die soziale Dynamik der Metropole, ihre Anziehungskraft für die Bergler und Provinzler sowie ihre Abwehrhaltung gegenüber den Migranten wird hier im Feld jener Erosionsmetaphorik gefasst, die Stifter in sei- 
nen Stadttexten noch als eine unter anderen erprobt, ${ }^{25}$ und ohne bis ins letzte Detail den Naturgesetzen zu folgen. Bemerkenswert ist dabei folgende Anschauung, ebenfalls vom Stephansturm aus:

Der Theil gerade zu unsern Füßen ist die eigentliche Stadt. Wir sehen sie, wie eine Scheibe um unsern Thurm herumliegen, ein Gewimmel und Geschiebe von Dächern, Giebeln, Schornsteinen, Thürmen, ein Durcheinanderliegen von Prismen, Würfeln, Piramiden, Parallelopipeden, Kuppeln, als sei das Alles in toller Kristallisation an einander geschossen, und starre nun da so fort. (HKG 9.1, S. IX)

Gipfelblick, Geschiebe und Kristallisation und also Übersicht, Erosionsbewegung und Starre gehen hier in eins zusammen, wenn auch im Modus des Konjunktivs und mit der distanzierenden Apposition des >Tollen Zusammenschließen des Differenten. Das weist schon auf die differenzierende Taxonomie des Nachsommers voraus, in dem die im Gipfelblick gewonnene Übersicht durch die Gesetzmäßigkeiten der Erosion in Frage gestellt und der Kristallzustand schon bei den ersten Feldversuchen nicht mehr das Maß aller Steine darstellen wird:

Allein da ich jezt meine Mineralien in der Gegend meines Aufenthaltes suchte, und zusammen trug, fand ich sie weit öfter in unkristallisirtem Zustande als in kristallisirtem, und sie zeigten da allerlei Eigenschaften für die Sinne, die sie dort nicht haben. (HKG 4.1, S. 33)

Heinrich beginnt darum, neben den auf Kristallisation beruhenden Mohs'schen Einteilungen, seine eigenen Beschreibungen zu machen. Ist er in seinen Erosionsbeobachtungen den großen Gesetzmäßigkeiten permanenter Bewegung auf der Spur, so sieht sich der Wien-Besucher der frühen Stadttexte angesichts des Geschiebes mit der Stillstellungsfigur der Starre konfrontiert.

In irritierendster Weise geschieht dies im Text Ein Gang durch die Katakomben, der den Blick vom Stephansturm herunter an eben demselben Ort um die Perspektive aus dem Erdinnern herauf ergänzt. Schon in der Prä-

25 Zentral ist in diesen frühen Stadttexten, wie Begemann gezeigt hat, die ozeanische Metaphorik; siehe Begemann, Die Welt der Zeichen (wie Anm. 2), S. 15-19. Diese kann zwar auch als Teil geologischer Prozesse verstanden werden, ist aber viel eher als Griff nach zeittypischen konventionellen Bildern erklärbar, denn vom Häusermeer schreiben viele andere auch. In Richtung Geologie weist jedoch das »Steinmeere«, das - ganz im Gegensatz zur eben noch als wogend beschriebenen Stadt - nun »starr« erscheint (HKG 9.1, S. VIII). Der allgegenwärtige Staub als festes Epitheton von Stadt und Straßen lässt sich gleichfalls erosiv lesen, ganz besonders aber sind im geologischen Kontext der Gipfelblick und die kartographische Übersicht zu verorten, die im Blick vom Stephansturm am Beispiel der Stadt erprobt werden. Vgl. dazu Schneider, Kulturerosionen (wie Anm. 7), S. 257f. Daneben aber stehen gleichberechtigt ganz andere Metaphernkomplexe, beispielsweise derjenige des Essens. 
ambel werden die Erosionsmetaphorik mit dem »Kiesel« und die erdgeschichtliche Perspektive mit der Zeitangabe von »Billionen Jahrtausende« als das Feld eingeführt, in der die Sache des Menschen verhandelt wird (HKG 9.1, S. 49f.). In der »unterirdische[n] Todtenstadt« (HKG 9.1, S. 52) begraben zu werden, war Privileg der Reichsten und Vornehmsten und mit der Erwartung ungestörter Totenruhe verbunden. Jetzt jedoch liegen die menschlichen Überreste auf Haufen, »starr, über einander geschichtet, eine werthlose, schauererregende Masse« (HKG 9.1, S. 54). Der Anblick dieser geschichteten Leichen, ihrer Teile und Knochen löst im Betrachter eine existentielle Erschütterung aus und verspricht auch aus dem Leser »ein[en] andere[n]« zu machen (HKG 9.1, S. 51).

In bestimmten Bereichen der Katakomben hat die Verwesung nur die Kleider, nicht aber die Körper der Toten affiziert, so dass diese nun nicht nur ihres sozialen Status', sondern auch ihrer Würde entblößt daliegen. Hier hätte das Naturgesetz erosiver Auflösung, in dessen Rahmen Menschliches verhandelt wird, etwas Tröstliches, doch die mumifizierten Körper mit ihren seit hunderten von Jahren im Todesaugenblick still gestellten Gesichtern scheinen nicht darunter fallen zu wollen, wie auch die Druckprobe mit dem Stock, die - analog zur Mohs'schen Ritzprobe am Mineral - an den Mumien vorgenommen wird, bestätigt. Es ist der Erzähler, der hier das widerständige Material über dessen vormalige Teilhabe an Kultur und über die Konjunktionsfunktion der Schrift in die allumfassende Erosion einbindet: »von dem Gebirge von Arbeiten aus dem Leben Dieser ist ein Blatt Geschichte übrig geblieben, und selbst dieses Blatt, wenn die Jahrhunderte rollen, schrumpft zu einer Zeile ein, bis auch endlich d i e s e verschwindet" (HKG 9.1, S. 60). Ob auch der Zeit-Ort des Einschiebsels Mensch in der Erdgeschichte zum Nichts strebt, ob auch die Erde selbst erodiert, »auf ewig aufhört « und »eine Leiche« wird - wie der Katakomben-Text explizit sinniert -, bleibt unentschieden, als Angstvorstellung aber im Staub und »namenlosen Mode $[\mathrm{r}] \ll$ der Totenstadt präsent (HKG 9.1, S. 58, 56).

Die semiotische Radikalität, mit der Stifter in seinen Texten der Erosionsbewegung bis hin zum einzelnen Sandkörnchen, Staubpartikel und noch darüber hinaus folgt, ist selbst im Geologie-besessenen 19. Jahrhundert außergewöhnlich. Sind geologische Beschreibungen und erdgeschichtliche Betrachtungen typische Bestandteile realistischer Raum-ZeitBezüge, so erscheinen sie doch meist eingebunden in ein textinternes Verweisungsnetz, das die Stellung des Menschen in Raum und Zeit stützt, sie nicht erschüttert. So beschreibt Theodor Fontane in seinen Wanderungen durch die Mark Brandenburg (1862-1889) die typischen Sandflächen der Gegend als landschaftliche Realitätspartikel und bindet sie in kulturhistorische Zusammenhänge ein, ohne dabei auf eine Tiefenzeit zu verweisen, 
die hinter historische Zeitrechnung zurück reichen würde. Wilhelm Raabes Stopfkuchen ist als Liebhaber-Paläontologe dagegen ein ausgewiesener Kenner der Erdgeschichte und erfolgreicher Sammler fossiler Funde. Die gefundenen Knochen wie auch die paläontologische Beschäftigung selbst stehen aber in so engen Verweisungszusammenhängen mit den Beziehungen der in die Mordgeschichte des Stopfleuchens (1890) involvierten Figuren, dass ihre Bezüge auf menschliche Geschichten der aktuellen Generation und derjenigen davor evident sind. Letztlich begrenzen auch die fossilen Funde selbst - es handelt sich insbesondere um Mammutknochen - die Öffnung auf die Tiefenzeit auf einen Rahmen, der auf die prähistorische Zeit verweist und die Bedeutung der Menschheitsgeschichte damit nicht in Frage stellt. Stifters erosives Raum-Zeit-Modell hingegen weist über die Angelegenheiten des Menschen hinaus in einer Radikalität, die selbst vor dem Planeten Erde nicht halt macht. 
Bereitgestellt von | UZH Hauptbibliothek / Zentralbibliothek Zürich Angemeldet Heruntergeladen am | 01.12.17 15:40 